\title{
Preferential water condensation on superhydrophobic nano-cones array
}

Yong Jin, Adnan Qamar, Yusuf Shi, and Peng Wang

Citation: Appl. Phys. Lett. 113, 211601 (2018); doi: 10.1063/1.5053697

View online: https://doi.org/10.1063/1.5053697

View Table of Contents: http://aip.scitation.org/toc/apl/113/21

Published by the American Institute of Physics

\section{Articles you may be interested in}

Phase control of plasmon enhanced two-photon photoluminescence in resonant gold nanoantennas

Applied Physics Letters 113, 211101 (2018); 10.1063/1.5051381

Efficient generation of nitrogen-vacancy center inside diamond with shortening of laser pulse duration Applied Physics Letters 113, 211102 (2018); 10.1063/1.5054730

Surfactant-induced chemical ordering of $\mathrm{GaAsN}: \mathrm{Bi}$

Applied Physics Letters 113, 211602 (2018); 10.1063/1.5045606

Atomic layer deposition of titanium nitride for quantum circuits

Applied Physics Letters 113, 212601 (2018); 10.1063/1.5053461

Beyond coffee ring: Anomalous self-assembly in evaporating nanofluid droplet on a sticky biomimetic substrate Applied Physics Letters 113, 213701 (2018); 10.1063/1.5063605

Nonlinear vibrations of circular single-layer black phosphorus resonators

Applied Physics Letters 113, 211901 (2018); 10.1063/1.5055950

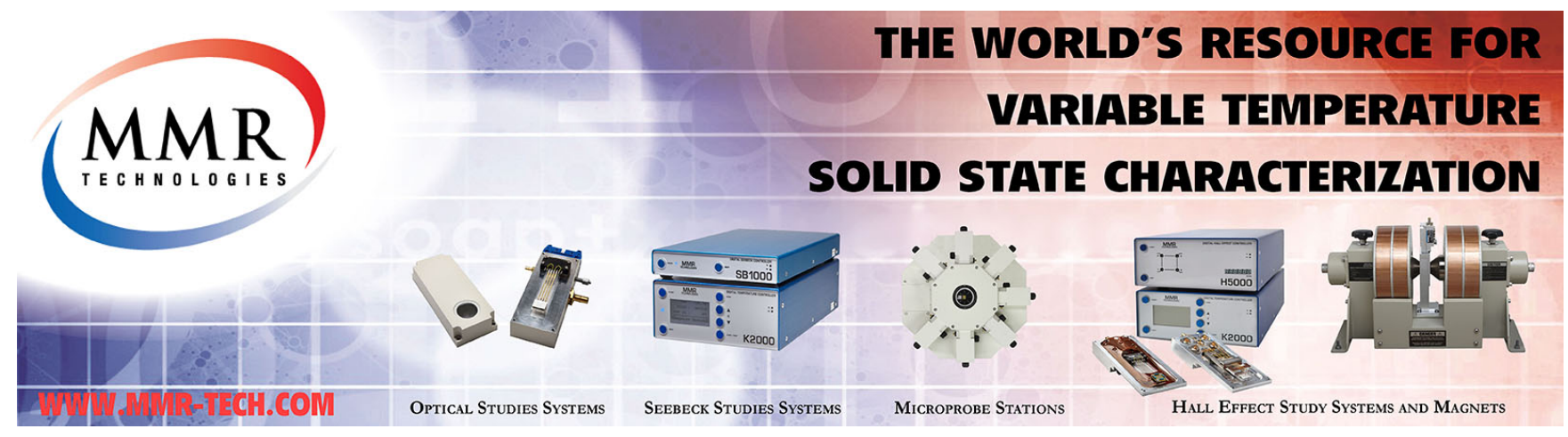




\title{
Preferential water condensation on superhydrophobic nano-cones array
}

\author{
Yong Jin, Adnan Qamar, Yusuf Shi, and Peng Wang ${ }^{\text {a) }}$ \\ Water Desalination and Reuse Center, Division of Biological and Environmental Science and Engineering, \\ King Abdullah University of Science and Technology, Thuwal 23955-6900, Saudi Arabia
}

(Received 24 August 2018; accepted 4 November 2018; published online 19 November 2018)

\begin{abstract}
Condensed water droplet jumping on superhydrophobic surfaces has been a hot research topic during the last decade for various applications including enhancing condensation, antifogging, self-cleaning, electrostatic energy harvesting, and anti-icing/frosting surfaces. In the present work, a superhydrophobic nano-cone array is prepared by simple chemical bath reaction followed by silane modification. The tip of an individual nano-cone is only around $10 \mathrm{~nm}$. The nano-cone array is also characterized by cavities among cones with varying sizes due to the imperfect alignment of individual nano-cones to the substrate. We demonstrate theoretically and experimentally that water condensation preferentially occurs within larger cavities among hydrophobized nano-cone arrays. After extrusion from cavities, droplets form a partial Cassie state which is a prerequisite of coalescence induced jumping. Moreover, growth rates of droplets extruded from larger cavities are larger than those from smaller cavities. Droplet jumping is observed at the very first coalescence and after multiple coalescence events under a microscope. The jumping droplet size is at tens of microns, demonstrating that droplets can be efficiently removed from the nano-cone array. In general, it is indicated that condensation can be controlled by purposely designing nano-scale structures, which would provide valuable suggestion for applications involving water condensation. Published by AIP Publishing.
\end{abstract}

https://doi.org/10.1063/1.5053697

Water condensation is a ubiquitous phenomenon occurring both in nature and engineered industrial processes with wide applications ranging from heat transfer, ${ }^{1}$ thermal desalination ${ }^{2-4}$ to dew harvesting. ${ }^{5-7}$ Dropwise condensation has been well recognized to be more efficient in terms of heat transfer than filmwise condensation ${ }^{8}$ and thus is preferred in such processes as condensation in power plant and water collection in membrane distillation. Recently, condensed droplet jumping on superhydrophobic surfaces has been a hot research topic during the last decade. ${ }^{9,10}$ Condensed water droplet jumping on superhydrophobic surfaces is an interesting phenomenon in which neighboring (two or multiple) condensed water droplets coalesce and jump off the surface due to the release of surface energy upon coalescence and low adhesion from the surface. Due to the small size of the jumping water droplets, enhanced condensation induced by droplet jumping has been reported at low sub-cooling degrees. ${ }^{11-20}$ In addition to enhancing condensation heat transfer, the droplet jumping phenomenon has also been reported to enhance the performance of antifogging, ${ }^{21}$ self-cleaning, ${ }^{22}$ electrostatic energy harvesting, ${ }^{23,24}$ thermal management of electronic devices, ${ }^{25,26}$ and anti-icing/frosting surfaces. ${ }^{27-31}$

It is critical to understand the condensation behavior on superhydrophobic surfaces for optimizing surface design in different applications. Although condensation on some well-patterned surfaces has been investigated using an environmental scanning electron spectroscope (E-SEM) ${ }^{32,33}$ and simulation studies, ${ }^{34}$ fundamental insights into condensation behavior on various existing superhydrophobic surfaces

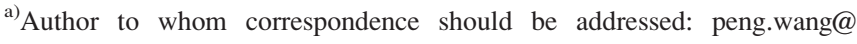
kaust.edu.sa
}

promoting condensation droplet jumping are still underexplored. $^{35-38}$

As a phase change process, water condensation starts from nucleation according to the classical nucleation theory, followed by individual droplet growth and coalescence. Water condensation spontaneously occurs when the change in Gibbs free energy $\left(\Delta \mathrm{G}_{\text {total }}\right)$ is negative. $\Delta \mathrm{G}_{\text {total }}$ can be expressed as

$$
\Delta \mathrm{G}_{\mathrm{total}}=-\rho V \Delta \mathrm{e}+\Delta \mathrm{G}_{\text {boundary }}
$$

where $\rho$ is the density of water, $\mathrm{V}$ is the volume of liquid water, $\Delta \mathrm{e}$ is the Gibbs free energy change from vapor to liquid per unit mass, and $\Delta \mathrm{G}_{\text {boundary }}$ is the boundary Gibbs free energy change including liquid-vapor and liquid solid interfaces. For homogeneous condensation, $\Delta \mathrm{G}_{\text {boundary }}$ only includes liquid vapor interfacial surface free energy, while it includes liquid-vapor and liquid-solid boundaries in heterogeneous condensation. Energy barrier needs to be overcome in order to form stable nuclei when a critical nuclei size $\left(\mathrm{r}^{*}\right.$, usually around $10 \mathrm{~nm}$ under low supersaturation conditions; see supplementary material $\mathrm{S} 1$ for a detailed description) is reached. ${ }^{39}$

The hydrophobic structure with a small dimension (characteristic length $\mathrm{R}$ ) comparable to the critical nuclei size $\left(\mathrm{r}^{*}\right)$ should have an impact on $\Delta \mathrm{G}_{\text {total }}$ as nucleation on extremely small objects resembles homogeneous condensation. ${ }^{40}$ Here, the normalized energy barrier is defined as the ratio of Gibbs free energy change of heterogeneous to that of homogeneous condensation when the critical nuclei size is reached. Figure 1(a) shows the normalized energy barrier as a function of ratio of structural dimension to critical nuclei size $\left(\mathrm{R} / \mathrm{r}^{*}\right)$ at different contact angles, which can be expressed as 

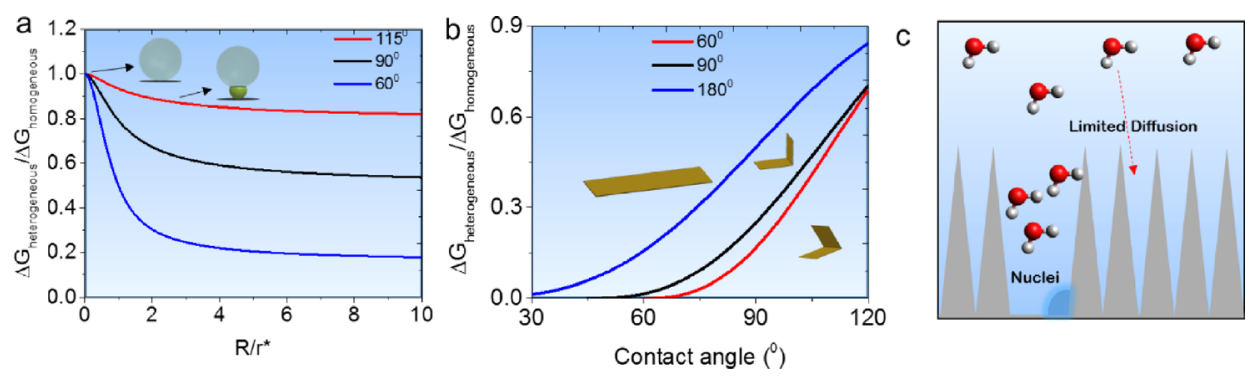

FIG. 1. (a) Relationship between the normalized nucleation energy barrier and the ratio of substrate dimension to critical nuclei size. (b) Relationship between the normalized nucleation energy barrier and the nano-substrate geometrical angle at different contact angles. (c) Sketch showing the limited diffusion of vapor into confined nano-structures. Note: the insets in Fig. 1(a) represent homogeneous condensation when $\mathrm{R}=0$ and heterogeneous condensation when the size is comparable to $r^{*}$.

$$
\begin{aligned}
\frac{\Delta G_{\text {heterogeneous }}^{*}}{\Delta G_{\text {homogeneous }}^{*}}= & \frac{1}{2}+\frac{1}{2}\left(\frac{1-R / r^{*} \cos \theta_{A}}{g}\right)^{3} \\
& +\frac{1}{2}\left(\frac{R}{r^{*}}\right)^{3}\left[2-3\left(\frac{\frac{R}{r^{*}}-\cos \theta_{A}}{g}\right)\right. \\
& \left.+\left(\frac{\frac{R}{r^{*}}-\cos \theta_{A}}{g}\right)^{3}\right]+\frac{3}{2} \cos \theta_{A}\left(\frac{R}{r^{*}}\right)^{2} \\
& \times\left(\frac{\frac{R}{r^{*}}-\cos \theta_{A}}{g}-1\right), \\
g= & \sqrt{1+\left(\frac{R}{r^{*}}\right)^{2}-2 \cos \theta_{A} \frac{R}{r^{*}},}
\end{aligned}
$$

where $\theta_{\mathrm{A}}$ is the advancing contact angle.

From Fig. 1(a), it can be inferred that nucleation is easy to occur on hydrophilic substrates and that nucleation is difficult to occur on the hydrophobic structure with a size dimension comparable to the critical nuclei size (i.e., $\mathrm{R} / \mathrm{r}^{*}$ close to 1; images in the inset of Fig. 1(a) representing homogeneous condensation when $\mathrm{R}=0$ and heterogeneous condensation when the size is comparable to $\left.\mathrm{r}^{*}\right)$. Thus, it can be deduced that nucleation can occur on a substrate with a larger dimension under low super saturation conditions more easily, which is similar to the easier occurrence of nucleation on hydrophilic sites. ${ }^{41}$ (see supplementary material $\mathrm{S} 1$ for detailed analysis).

Moreover, nucleation is also affected by the wedge-like structure between two nanostructures (Fig. S2 in supplementary material S1). ${ }^{37,42}$ The normalized energy barrier of nucleation in the wedge-like structure is expressed as

$$
\begin{aligned}
\frac{\Delta G_{\text {heterogeneous }}^{*}}{\Delta G_{\text {homogeneous }}^{*}}= & \frac{1}{4 \pi}\left[\cos \theta_{A} \sin \theta_{A}^{2} \sin \varepsilon-\cos \theta_{A}\left(3-\cos \theta_{A}{ }^{2}\right) \varepsilon\right. \\
+ & \left.4 \sin ^{-1}\left(\sin \frac{\varepsilon}{2} \sin \frac{\chi}{2}\right)\right] \\
& \cos \frac{\varepsilon}{2}=\cot \theta_{A} \cot \frac{\chi}{2}
\end{aligned}
$$

where $\theta_{\mathrm{A}}$ is the advancing contact angle and $\chi$ is the angle of the wedge.

Figure 1(b) shows the normalized Gibbs free energy barrier for nucleation to occur on wedges of different angles

$\left[60^{\circ}, 90^{\circ}\right.$, and $180^{\circ}$; see the images in the inset of Fig. 1(b)]. It shows that heterogeneous condensation should be usually more easily observed than the homogeneous one, especially in cavity-like structures. For example, the nucleation energy barrier within the $60^{\circ}$ wedge is much lower than that on the flat surface $\left(180^{\circ}\right.$ wedge $)$.

In addition, as sketched in Fig. 1(c), water vapor can penetrate through larger cavities among nanostructures more easily than small cavities. Simulation and experimental works showed that the nanostructure assembly has an impact on vapor diffusion, which further impacts water condensation. ${ }^{15,34}$ For example, it was shown that condensation tended to occur on the top of nanostructures with a high length to inter-distance ratio. ${ }^{34}$

We test our above analysis by conducting condensation on the nano-cone array prepared from $\mathrm{Co}(\mathrm{OH})_{2}$ deposition on the substrate (see supplementary material S2 for the preparation method). Figures 2(a) and 2(b) show the top view and cross-sectional view SEM images of the as-grown nano-cone array. It is observed that the nano-cones are closely packed together. Another important characteristic of the closely
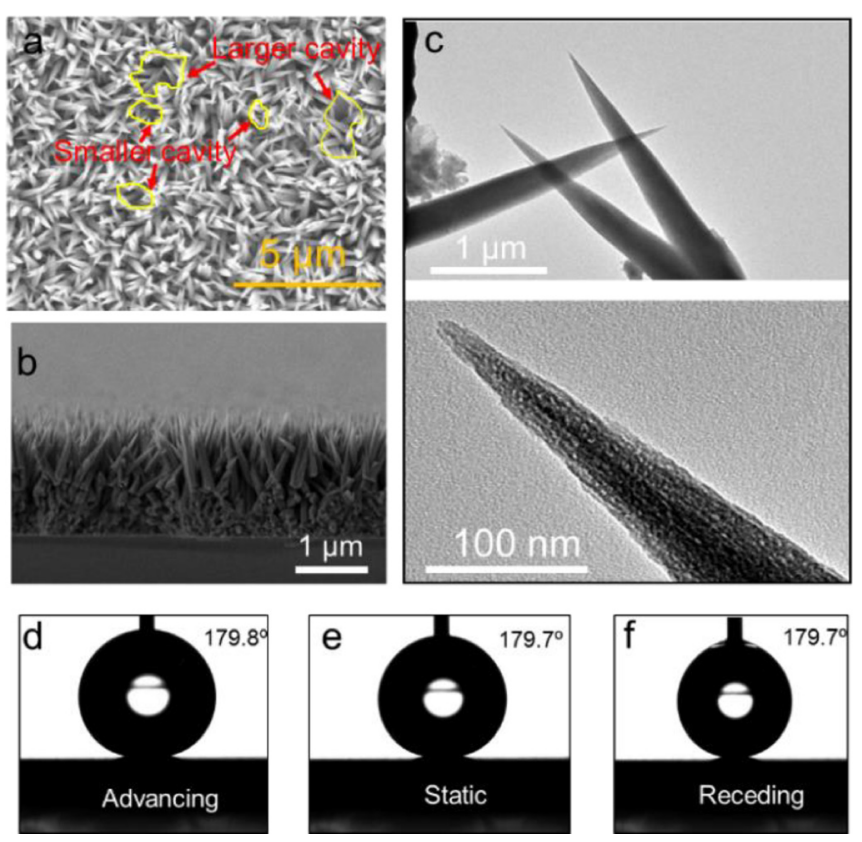

FIG. 2. (a) Top view and (b) cross-sectional view SEM images of the asprepared nano-cone array. (c) TEM images of an individual nano-cone. (d) Advancing, (e) static, and (f) receding contact angles of the as-prepared nano-cone array after the hydrophobic coating modification. 
packed nano-cone array is that single nano-cone is not always well aligned perpendicularly to the substrate. The majority of the nano-cones are slanted to different extents, giving rise to cavities with varying sizes among nano-cones [delineated by the yellow circled lines in Fig. 2(a)]. These cavities with different sizes are very important in the following condensation experiment. From both Fig. 2(b) (SEM) and Fig. 2(c) (TEM), the length, tip diameter, and base diameter of a typical nano-cone are determined to be $2 \mu \mathrm{m}, 10 \mathrm{~nm}$, and $350 \mathrm{~nm}$, respectively. A monolayer of a hydrophobic coating (perfluoro-silane) is then deposited on the nano-cones by a simple chemical vapor deposition (CVD) method. After modification, the advancing, static, and receding contact angles of the sample are $179.8^{\circ}, 179.7^{\circ}$, and $179.7^{\circ}$, respectively [Figs. 2(d)-2(f)], indicating a superhydrophobic state with an extremely small hysteresis.

A water condensation experiment under an environmental SEM (E-SEM) is then conducted to observe the condensation processes down to microscale on the as-prepared superhydrophobic nano-cone array. Both side and top views of single droplet growth in Fig. 3(a) show that the first step of an individual droplet growth starts from filling a cavity with certain size among nano-cones. Then, water inside the cavities starts to extrude upwards, forming a spherical droplet on the surface. The apparent contact angle of the spherical droplet is almost $180^{\circ}$. From Fig. 3(a), a partial Cassie state is clearly observed, which is a prerequisite for coalescence a

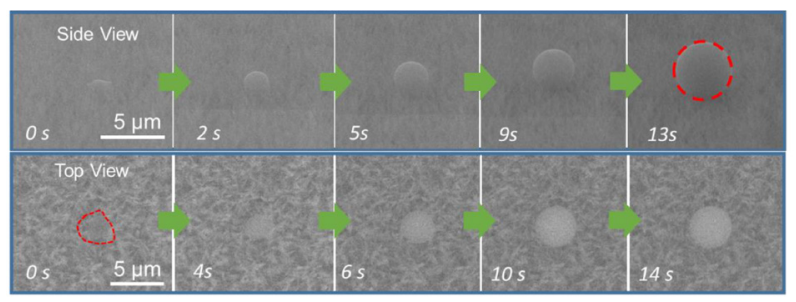

b

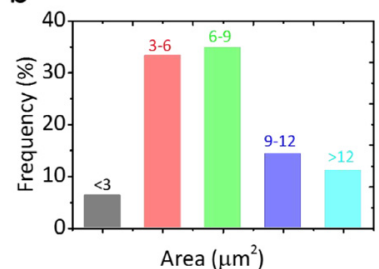

d

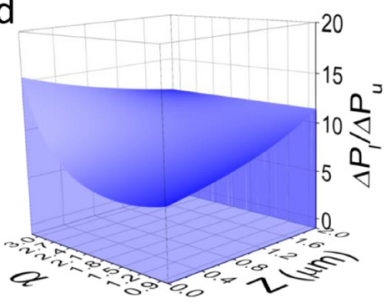

C

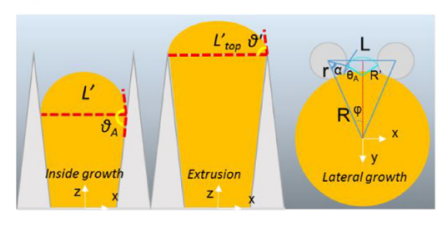

e

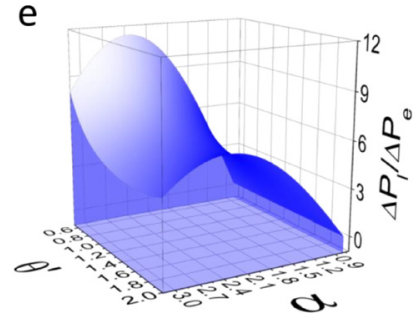

FIG. 3. (a) E-SEM images showing the side view and top view of the growth process of individual droplets on the nano-cone array. (b) Bar graph showing the distribution frequency of the cavity area where the water film extrudes from. (c) Schematic showing the contact line frontier of lateral and upward growth in larger cavities. (d) Figure showing the ratio of lateral growth Laplace pressure $\left(\Delta \mathrm{P}_{1}\right)$ to upward growth Laplace pressure $\left(\Delta \mathrm{P}_{\mathrm{u}}\right)$ as a function of parameters $\alpha$ and Z. (e) Figure showing the ratio of lateral growth Laplace pressure $\left(\Delta \mathrm{P}_{1}\right)$ to upward growth Laplace pressure $\left(\Delta \mathrm{P}_{\mathrm{e}}\right)$ as a function of parameters $\alpha$ and $\theta^{\prime}$. induced jumping. During E-SEM experiments, it is found that droplets initially appearing are always in the partial Cassie state similar to the droplet shown in Fig. 3(a) (Fig. S4). The benefit of the partial Cassie state over the complete Cassie state is proved previously to facilitate faster individual droplet growth. ${ }^{43}$ A statistical analysis of the size distribution of the water filled cavities is shown in Fig. 3(b), indicating that the area of the filled cavities mostly lies in the range of $3-9 \mu \mathrm{m}^{2}$, which is far beyond the areas of small cavities. These results indicate that droplets preferentially grow from large cavities on our prepared surface. The phenomenon is well correlated with the theory discussed above. Due to the small dimension and hydrophobic nature (the advancing contact angle is $115^{\circ}$; Fig. S5) of nano-cone tips, it is difficult for nucleation to occur on the top of the nano-cone array under low supersaturation conditions such as the present E-SEM experiment (cooling stage temperature: $-2{ }^{\circ} \mathrm{C}$; vapor pressure: around $600 \mathrm{~Pa}$ ). Meanwhile, wedge structures at the bottom of larger cavities give rise to a lower nucleation energy barrier and easy diffusion. Thus, it seems that nucleation prefers to occur at the bottom side of larger cavities.

After formation of stable nuclei, individual droplets start to grow within the cavities. The growth direction of individual droplets within the cavities is limited by the Laplace pressure difference. The nano-cone array is characterized by larger cavities surrounded by closely packed nano-cones. Thus, we reasonably establish a simplified model to study the growth direction of water inside the cavities. The model structure consists of 36 closely packed individual nanocones forming a circular large cavity (Fig. S6). Figure 3(c) shows water growth (in orange) inside the model structure both from the side view (upward growth and extrusion) and top view (lateral growth). The liquid advancing front needs to overcome Laplace pressure to squeeze into the space between two neighboring nano-cones in order to grow laterally. As is shown in Fig. 3(c), the lateral growth of droplets needs to overcome the Laplace pressure induced by curvature which can be expressed as

$$
\Delta P_{l}=\frac{2 \gamma}{\frac{\frac{L}{2}+[1-\sin (\alpha+\varphi)] r}{\sin \left(\theta_{A}+\alpha+\varphi-\pi\right)}} .
$$

The liquid front contains a Laplace pressure defined by the temporal cavity size when the liquid front moves upwards. Upward growth inside cavities needs to overcome Laplace pressure

$$
\Delta P_{u}=\frac{2 \gamma}{\frac{L^{\prime} / 2}{\cos (\pi-\beta / 2-\theta \mathrm{A})}} .
$$

Extrusion out of cavity needs to satisfy the condition where $\theta^{\prime}$ is larger than the advancing contact angle $\left(\theta_{\mathrm{A}}\right)$. Upward extrusion needs to overcome the Laplace pressure which can be expressed as

$$
\Delta P_{e}=\frac{2 \gamma}{\frac{L_{t o p}^{\prime}}{2} / \sin \theta^{\prime}}
$$


The value of $\Delta \mathrm{P}_{1}, \Delta \mathrm{P}_{\mathrm{u}}$, and $\Delta \mathrm{P}_{\mathrm{e}}$ at the same moment should be compared in order to predict the growth direction. If $\Delta \mathrm{P}_{1}$ $>\Delta \mathrm{P}_{\mathrm{u}}$, upward growth inside cavities is favorable. If $\Delta \mathrm{P}_{1}$ $>\Delta \mathrm{P}_{\mathrm{e}}$, upward extrusion is favorable. Figure 3(d) shows the plot of $\Delta \mathrm{P}_{1} / \Delta \mathrm{P}_{\mathrm{u}}$ as a function of angle $\alpha$ and height $\mathrm{z}$ as sketched in Fig. 3(c). It is clear that $\Delta \mathrm{P}_{1}$ is larger than $\Delta \mathrm{P}_{\mathrm{u}}$ for any given $\alpha$ and $\mathrm{z}$. Figure 3(e) shows the plot of $\Delta \mathrm{P}_{1} / \Delta \mathrm{P}_{\mathrm{e}}$ as a function of angle $\alpha$ and $\theta^{\prime}$ as sketched in Fig. 3(c). In order to grow laterally, angle $\alpha$ needs to be larger than $\pi$. However, $\Delta \mathrm{P}_{1} / \Delta \mathrm{P}_{\mathrm{e}}$ increases with increasing $\alpha$, resulting in values larger than 1 before angle $\alpha$ reaches $\pi$. Thus, it is clear that upward growth and extrusion are favorable (see supplementary material S5 for detailed analysis).

Figure 4(a) shows typical E-SEM images of droplet growth with time. From the images, droplets of different sizes exist before the very first coalescence event occurs. Interspace among droplets is at the magnitude of $10 \mu \mathrm{m}$ under present supersaturation conditions. The relationship between the cavity size and the mass growth rate of typical droplets 1-7 marked by the red number in Fig. 4(a) is shown in Fig. 4(b). It is obvious that the mass growth rate is almost linearly proportional to the cavity area. The mass growth rate of droplets corresponds to the heat flux transferred. The faster growth rate within larger cavities indicates larger heat flux transferred. From the image shown in the inset of Fig. 4(b), it is found that larger cavities provide a larger heat transfer area from both surrounding solid nano-cones and liquid within cavities than smaller cavities.

The condensation experiment in the ambient environment (sub cooling degree: $6{ }^{\circ} \mathrm{C}$; supplementary material S6)
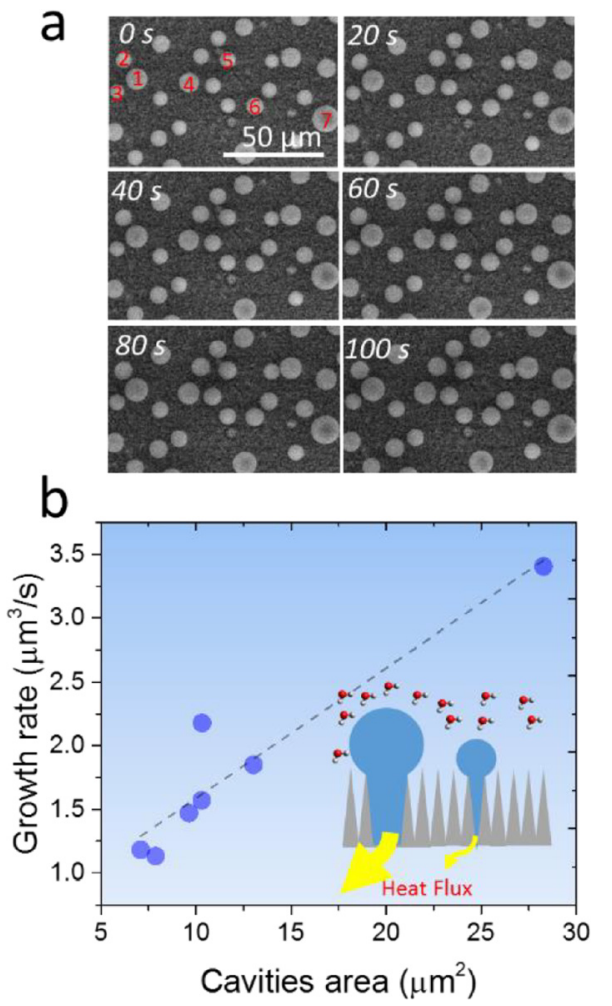

FIG. 4. (a) E-SEM iamges showing droplet growth with time before the first coalescence event occurs. (b) Relationship between the cavity area and the droplet mass growth rate (with the image in the inset showing higher heat flux from larger cavities). is also conducted on the superhydrophobic nano-cone array. Observation under an optical microscope shows that the droplets initiate their first coalescence at a diameter of around $6.5 \mu \mathrm{m}$ [Fig. 5(a), red circle]. At low supersaturation conditions such as the present experiment, the nucleation density is at the magnitude of $10^{5} / \mathrm{mm}^{2}$. Thus, the first droplet coalescence normally occurs when the droplet size is around several microns. Coalescence induced droplet jumping indeed occurs on the surface with droplets disappearing from the view after coalescence. It is observed that droplet jumping can occur when the very first coalescence event happens including two droplet and multi-droplet coalescence [Fig. 5(a), red circle]. Jumping also occurs following two or more coalescence events [Fig. 5(a), orange circle]. Although it is impossible for us to get the jumping velocity of droplets from the very first coalescence due to the resolution limit, it should be affected by adhesion force from cavities, which would result in lower jumping velocity compared to previous predictions. ${ }^{44}$ Typical high speed camera images in Fig. 5(b) confirm two droplet coalescence induced jumping after multiple coalescence (the droplet size is around $12 \mu \mathrm{m}$ ). A three-dimensional Volume of Fluid (VOF) simulation (supplementary material S7) of two spherical drops on a flat superhydrophobic surface with a contact angle of $179.7^{\circ}$ is also performed. The velocity of jumping after multiple coalescence events is well correlated by previous predictions ${ }^{44}$ (supplementary material S6) and our own VOF simulation [Fig. 5(c), Fig. S9] because droplets from multiple coalescence are at a complete Cassie state.

In conclusion, we theoretically and experimentally demonstrate that condensation preferentially occurs within larger cavities among nano-cones and forms a partial Cassie state. Growth rates of droplets from larger cavities are larger than those from smaller cavities due to larger heat transfer

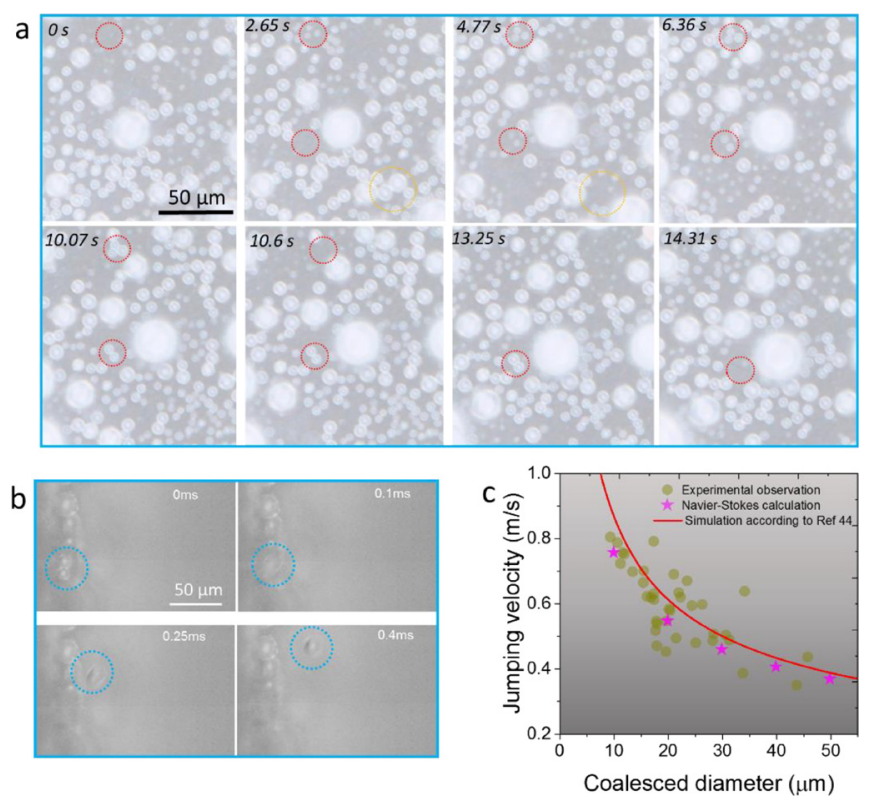

FIG. 5. (a) Microscopy images showing first coalescence induced jumping (red circles) and jumping after multiple coalescence (yellow circles). (b) High speed camera images showing two droplet coalescence induced jumping after multiple coalescence events. (c) Relationship between the initial droplet jumping velocities and coalesced jumping droplet diameters. 
interfaces. Meanwhile, efficient removal of droplets by coalescence induced jumping (at tens of microns) is observed.

See supplementary material for all theoretical analysis and experimental details.

We are grateful to KAUST for very generous financial support. We thank Sankara Arunachalam for helping setting up high speed camera equipment.

${ }^{1}$ J. W. Rose, Heat Mass Transfer 35, 479 (1999).

${ }^{2}$ G. Amy, N. Ghaffour, Z. Li, L. Francis, R. V. Linares, T. Missimer, and S. Lattemann, Desalination 401, 16 (2017).

${ }^{3}$ T. Mezher, H. Fath, Z. Abbas, and A. Khaled, Desalination 266, 263 (2011).

${ }^{4}$ A. Al-Karaghouli and L. L. Kazmerski, Renewable Sustainable Energy Rev. 24, 343 (2013).

${ }^{5}$ A. Lee, M.-W. Moon, H. Lim, W.-D. Kim, and H.-Y. Kim, Langmuir 28, 10183 (2012)

${ }^{6}$ D. Seo, J. Lee, C. Lee, and Y. Nam, Sci. Rep. 6, 24276 (2016).

${ }^{7}$ Y. Jin, L. Zhang, and P. Wang, Global Challenges 1, 1700019 (2017).

${ }^{8}$ E. Schmidt, W. Schurig, and W. Sellschopp, Tech. Mech. Thermodyn. 1, 53 (1930) [in German].

${ }^{9}$ C.-H. Chen, Q. Cai, C. Tsai, C.-L. Chen, G. Xiong, Y. Yu, and Z. Ren, Appl. Phys. Lett. 90, 173108 (2007).

${ }^{10}$ J. B. Boreyko and C.-H. Chen, Phys. Rev. Lett. 103, 184501 (2009).

${ }^{11}$ R. Wen, S. Xu, D. Zhao, Y.-C. Lee, X. Ma, and R. Yang, ACS Appl. Mater. Interfaces 9, 44911 (2017).

${ }^{12}$ Y. Hou, M. Yu, X. Chen, Z. Wang, and S. Yao, ACS Nano 9, 71 (2015).

${ }^{13}$ N. Miljkovic, D. J. Preston, R. Enright, and E. N. Wang, ACS Nano 7, 11043 (2013).

${ }^{14}$ D. J. Preston and E. N. Wang, Joule 2, 205 (2018).

${ }^{15}$ R. Wen, S. Xu, X. Ma, Y.-C. Lee, and R. Yang, Joule 2, 269 (2018).

${ }^{16} \mathrm{R}$. Wen, Q. Li, J. Wu, G. Wu, W. Wang, Y. Chen, X. Ma, D. Zhao, and R. Yang, Nano Energy 33, 177 (2017).

${ }^{17}$ N. Miljkovic, R. Enright, Y. Nam, K. Lopez, N. Dou, J. Sack, and E. N. Wang, Nano Lett. 13, 179 (2013).

${ }^{18}$ C. Xuemei, W. Jun, M. Ruiyuan, H. Meng, K. Nikhil, Y. Shuhuai, and W. Zuankai, Adv. Funct. Mater. 21, 4617-4623 (2011).

${ }^{19}$ N. Miljkovic and E. N. Wang, MRS Bull. 38, 397-406 (2013).
${ }^{20}$ R. Wang, J. Zhu, K. Meng, H. Wang, T. Deng, X. Gao, and L. Jiang, Adv. Funct. Mater. 29, 1800634 (2018).

${ }^{21}$ T. Mouterde, G. Lehoucq, S. Xavier, A. Checco, C. T. Black, A. Rahman, T. Midavaine, C. Clanet, and D. Quéré, Nat. Mater. 16, 658 (2017).

${ }^{22}$ K. M. Wisdom, J. A. Watson, X. Qu, F. Liu, G. S. Watson, and C.-H. Chen, Proc. Natl. Acad. Sci. U. S. A. 110, 7992 (2013).

${ }^{23}$ N. Miljkovic, D. J. Preston, R. Enright, and E. N. Wang, Nat. Commun. 4, 2517 (2013).

${ }^{24}$ N. Miljkovic, D. J. Preston, R. Enright, and E. N. Wang, Appl. Phys. Lett. 105, 013111 (2014).

${ }^{25}$ J. B. Boreyko, Y. Zhao, and C.-H. Chen, Appl. Phys. Lett. 99, 234105 (2011).

${ }^{26}$ J. Oh, P. Birbarah, T. Foulkes, S. L. Yin, M. Rentauskas, J. Neely, R. C. N. Pilawa-Podgurski, and N. Miljkovic, Appl. Phys. Lett. 110, 123107 (2017).

${ }^{27}$ A. Kim, C. Lee, H. Kim, and J. Kim, ACS Appl. Mater. Interfaces 7, 7206 (2015).

${ }^{28}$ K. R. Murphy, W. T. McClintic, K. C. Lester, C. P. Collier, and J. B. Boreyko, ACS Appl. Mater. Interfaces 9, 24308 (2017).

${ }^{29}$ J. B. Boreyko and C. P. Collier, ACS Nano 7, 1618 (2013).

${ }^{30}$ M. J. Kreder, J. Alvarenga, P. Kim, and J. Aizenberg, Nat. Rev. Mater. 1, 15003 (2016).

${ }^{31}$ Q. Zhang, M. He, J. Chen, J. Wang, Y. Song, and L. Jiang, Chem. Commun. 49, 4516 (2013).

${ }^{32}$ C. Dorrer and J. Rühe, Langmuir 23, 3820 (2007).

${ }^{33}$ R. Enright, N. Miljkovic, A. Al-Obeidi, C. V. Thompson, and E. N. Wang, Langmuir 28, 14424 (2012).

${ }^{34}$ Q. Zhang, D. Sun, Y. Zhang, and M. Zhu, Langmuir 30, 12559 (2014).

${ }^{35}$ M. D. Mulroe, B. R. Srijanto, S. F. Ahmadi, C. P. Collier, and J. B. Boreyko, ACS Nano 11, 8499 (2017).

${ }^{36}$ C. S. Sharma, J. Combe, M. Giger, T. Emmerich, and D. Poulikakos, ACS Nano 11, 1673 (2017).

${ }^{37}$ A. Aili, Q. Ge, and T. Zhang, J. Heat Transfer 139, 112401 (2017).

${ }^{38}$ A. Aili, H. Li, M. H. Alhosani, and T. Zhang, ACS Appl. Mater. Interfaces 8, 21776 (2016).

${ }^{39}$ D. Kashchiev, Nucleation: Basic Theory with Applications (ButterworthHeinemann, Oxford, 2000).

${ }^{40}$ N. H. Fletcher, J. Chem. Phys. 29, 572 (1958).

${ }^{41}$ K. K. Varanasi, M. Hsu, N. Bhate, W. Yang, and T. Deng, Appl. Phys. Lett. 95, 094101 (2009).

${ }^{42}$ C. A. Sholl and N. H. Fletcher, Acta Metall. 18, 1083 (1970).

${ }^{43}$ N. Miljkovic, R. Enright, and E. N. Wang, ACS Nano 6, 1776 (2012).

${ }^{44}$ R. Enright, N. Miljkovic, J. Sprittles, K. Nolan, R. Mitchell, and E. N. Wang, ACS Nano 8, 10352 (2014). 